\title{
Modeling Elasticity in Crystal Growth
}

\author{
K. R. Elder, ${ }^{1}$ Mark Katakowski, ${ }^{1}$ Mikko Haataja, ${ }^{2}$ and Martin Grant ${ }^{2}$ \\ ${ }^{1}$ Department of Physics, Oakland University, Rochester, Michigan 48309-4487 \\ ${ }_{2}^{2}$ Physics Department, Rutherford Building, 3600 rue University, McGill University, Montréal, Québec, Canada H3A $2 T 8$
}

(Received 7 July 2001; published 4 June 2002)

\begin{abstract}
A new model of crystal growth is presented that describes the phenomena on atomic length and diffusive time scales. The former incorporates elastic and plastic deformation in a natural manner, and the latter enables access to time scales much larger than conventional atomic methods. The model is shown to be consistent with the predictions of Read and Shockley for grain boundary energy, and Matthews and Blakeslee for misfit dislocations in epitaxial growth.
\end{abstract}

DOI: $10.1103 /$ PhysRevLett.88.245701

The appearance and growth of crystal phases occurs in many technologically important processes including epitaxial growth and zone refinement. While a plethora of models have been constructed to examine various aspects of these phenomena, it has proven difficult to develop a computationally efficient model that can be used for a wide range of applications. For example, standard molecular dynamics simulations include the necessary physics but are limited by atomic sizes $(\AA)$ and phonon time scales (ps). Conversely, continuum field theories can access longer length (i.e., correlation length) and time (i.e., diffusive) scales, but are difficult to incorporate with the appropriate physics. In this paper a new model is presented that includes the essential physics and is not limited by atomic time scales.

To illustrate the features that must be incorporated, it is useful to consider two examples. First, consider the nucleation and growth of crystals from a pure supercooled liquid or vapor phase. In such a process, small crystallites nucleate (heterogeneously or homogeneously) and grow in arbitrary locations and orientations. Eventually, the crystallites impinge on one another and grain boundaries are formed. Further growth is then determined by the evolution of grain boundaries. Now consider the growth of a thin crystal film on a substrate of a different crystal structure. The substrate stresses the overlying film which can destabilize the growing film and cause an elastic defect-free morphological deformation [1,2], plastic deformation involving misfit dislocations [3], or a combination of both. Thus, the model must be able to nucleate crystallites at arbitrary locations and orientations and contain elastic and plastic deformations. While all these features are naturally incorporated in atomistic descriptions, they are much more difficult to include in continuum or phase field models.

Historically, many continuum models have been developed to describe certain aspects of crystal growth and liquid/solid transitions in general. At the simplest level, "model A" in the Halperin and Hohenberg [4] classification scheme has been used to describe liquid/solid transitions. This model treats all solids equivalently and does not introduce any crystal anisotropy. Extensions to this basic
PACS numbers: 64.60.Cn, 05.70.Ln, 64.60.My, 81.30.Hd

model have been developed to incorporate a solid phase that has multiple states that represent multiple orientations $[5,6]$ or, recently [7], an infinite number of orientations. Unfortunately, these models do not properly include plastic and elastic deformations. Other models [8-12] have sought to include elastic and plastic deformations, but cannot account for multiple orientations.

In this work, these limitations are overcome by considering a free energy that is minimized by a periodic hexagonal (i.e., solid) state. Such free energies have arisen in many other physical systems [13,14] (such as water/ surfactant systems, copolymers, Rayleigh-Bénard convection, and ferromagnetic films) and in some instances have even been described in terms of crystalline terminology [14]. The model used in this work describes the statics and dynamics of a conserved field, $\psi$, by the following free energy and equation of motion:

$$
\mathcal{F}=\int d \vec{r}\left\{\psi\left[\left(q_{o}^{2}+\nabla^{2}\right)^{2}-\epsilon\right] \psi / 2+\psi^{4} / 4\right\}
$$

and

$$
\partial \psi / \partial t=\nabla^{2}(\delta \mathcal{F} / \delta \psi)+\eta,
$$

where $\eta$ is a stochastic noise, with zero mean and correlations $\left\langle\eta(\vec{r}, t) \eta\left(\vec{r}^{\prime}, t^{\prime}\right)\right\rangle=-G \nabla^{2} \delta\left(\vec{r}-\vec{r}^{\prime}\right) \delta\left(t-t^{\prime}\right)$, and $G=0$ hereafter, $q_{o}$ and $\epsilon$ are constants. The field $\psi$ represents the local mass density. The specific free energy given in Eq. (1) was chosen as it is the simplest form that produces periodic states. The simplicity is important, as it leads to computationally efficient numerical schemes. Producing periodic structures is crucial, as such states naturally allow for elastic and plastic deformations, multiple orientations, and can accommodate free surfaces, as required for a crystal phase. The dynamical equation of motion, Eq. (2), was chosen to conserve the local mass density.

The focus of this paper is on two dimensions (2D); it is straightforward to extend these calculations to 3D. In 2D, this free energy is minimized by striped $\left(\psi_{s}\right)$, hexagonal $\left(\psi_{h}\right)$, and constant $\left(\psi_{c}\right)$ states depending on the average value, $\bar{\psi}$, of $\psi$. To estimate the phase diagram, these states 
can be approximated by $\psi_{s}=A_{s} \sin \left(q_{s} x\right)+\bar{\psi}, \quad \psi_{h}=$ $A_{h}\left[\cos \left(q_{h} x\right) \cos \left(q_{h} y / \sqrt{3}\right)+\cos \left(2 q_{h} y / \sqrt{3}\right) / 2\right]+\bar{\psi}$, and $\psi_{c}=\bar{\psi}$. Substituting these expressions into Eq. (1) and minimizing subject to the constraint $\int d \vec{r} \psi=\bar{\psi}$ gives the values for the characteristic amplitudes $A$ and wave number $q$ [15] and the phase diagram shown in Fig. 1a.

The linear elastic properties of the hexagonal phase can be determined by calculating the change of energy [as described by Eq. (1)] under shear, bulk, or dilational deformations within the approximation given for $\psi_{h}$ above. For example, the energy for a compression or expansion can be estimated by substituting Eq. (3) into Eq. (1), minimizing Eq. (1) with respect to $A_{h}$, and then determining the change in $\mathcal{F}$ as a function of $q_{h}$, since $q_{h}$ is inversely proportional to the periodicity or lattice spacing. This change in energy can then be related to elastic constants in the standard fashion [16]. Using this method, it is straightforward to find the elastic moduli for the isotropic solid. For the free energy given in Eq. (1), the results are $C_{12}=C_{44}=C_{11} / 3$, where $C_{12}=\left[\left(3 \bar{\psi}+\sqrt{15 \epsilon-36 \bar{\psi}^{2}}\right) q_{o}^{2}\right]^{2} / 75$, which are consistent with an isotropic solid. For these coefficients [17], the Poisson ratio is $\nu=1 / 3$ and the shear modulus is $\mu=C_{44}$.

The energy per unit surface length, $E_{L}$, between grains that differ in orientation by an angle $\theta$ was determined numerically and, in Fig. 1b, compared with the prediction of Read and Shockley [18], i.e., $E_{L}=E_{M} \theta\left[1-\ln \left(\theta / \theta_{M}\right)\right]$, where $E_{M}$ and $\theta_{M}$ are constants. The parameters of the simulations were $\left(\epsilon, \bar{\psi}, q_{o}\right)=(4 / 15,1 / 4,1)$. In all the
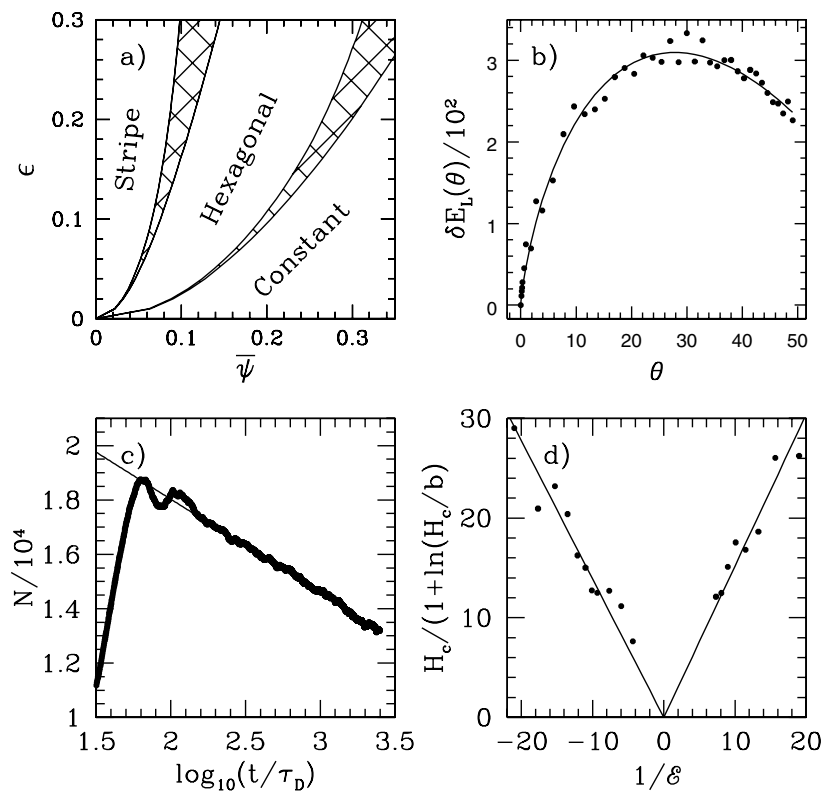

FIG. 1. (a) Mean field phase diagram. In this figure the "hatched" regions are coexistence regions. (b) Grain boundary energy. The points are from numerical simulations and the line a fit to the Read-Shockley equation. (c) Grain growth. In this figure, the number defects is plotted and the solid line is a guide to the eye. (d) Epitaxial growth. The points are from numerical simulations and the lines are best fits. simulations conducted, the time and space size were $\Delta t=$ 0.01 and $\Delta x=\pi / 4$, respectively. The Read-Shockley form closely fits the data for $\theta_{M}=27.85^{\circ}$ and $E_{M}=$ 0.064 . The maximum angle $\theta_{M}$ is similar to those observed in experiment [18]. The maximum energy/length, $E_{M}$, can be estimated using the Read-Shockley equation [18] in 2D [17] using the elastic constants estimated above, $E_{M}=\mu(1+\nu) b / 4 \pi=C_{12} b / 3 \pi$, where $b=2 \pi / q_{h}$ is the magnitude of the Burgers vector. For the parameters used in the simulations, this gives $E_{M}=0.044$, as compared to our measured value of 0.064 . This is a reasonable result considering the inherent approximations in the Read-Shockley equation and the approximate form chosen for $\psi_{h}$ used in calculating the elastic constants.

The main advantage of the current approach over molecular dynamics simulations is the time scales that are accessible. To illustrate this point, it is useful to calculate the diffusion time using a standard Bloch-Floquét linear analysis. In such an approach, the dynamics of a perturbation $(\delta \psi)$ around an equilibrium crystal state $\left(\psi_{\text {eq }}\right)$ is obtained by first substituting $\psi=\delta \psi+\psi_{\text {eq }}$ into Eq. (2) and linearizing in $\delta \psi$. Substituting appropriate forms for the equilibrium state, i.e., $\psi_{\mathrm{eq}}=\bar{\psi}+$ $\sum a_{n, m} \exp \left[i\left(n q_{x} x+m q_{y} y\right)\right]$, and perturbation along one of the three principal axes, i.e., $\delta \psi=\sum \delta a_{n, m}(t) \times$ $\exp \left\{i\left[\left(n q_{x}+Q\right) x+m q_{y} y\right]\right\}, \quad$ and integrating over $\exp \left[i\left(k q_{x} x+l q_{y} y\right)\right]$ gives an equation of motion for the modes $\delta a_{n, m}$. Using the approximation for $\psi_{\text {eq }}$ given by $\psi_{h}$ and four modes $\delta a_{ \pm 1, \pm 1}$ for the perturbation, the resulting equations can be solved analytically, assuming $\delta a_{n, m} \sim \exp (-\omega t)$. The smallest eigenvalue is equal to $\omega=3 q_{o}^{4} Q^{2}$ implying a diffusion constant of $3 q_{o}^{4}$. In terms of time steps, this implies it takes roughly 1000 time steps for a diffusion time, $\tau_{D}$ [19], for the simulation parameters used in this paper.

The ability of the continuum model to describe multiple crystals in arbitrary orientations and locations with the appropriate grain boundary energies on diffusive time scales makes it ideal for the study of grain boundary growth. To study this phenomenon, a system of size $4096 \Delta x \times$ $4096 \Delta x$ was simulated with parameters $\left(\epsilon, \bar{\psi}, q_{o}\right)=$ $(0.1,0.05,1)$. To begin the simulations, 256 crystals were nucleated at arbitrary locations by placing large fluctuations in $\psi$ at each nucleation site. As time evolves, the small crystallites grow from the initial seeds until impingement. Eventually, the entire system is filled with the hexagonal phase, and further evolution continues by motion at grain boundaries. A portion of a sample configuration is given in Fig. 2. At this stage in the simulations, there were approximately 236000 particles. To study the subsequent dynamics, the number of defects, where a defect is defined as a particle that does not have six nearest neighbors, was monitored. The number of defects is shown in Fig. 1c as a function of the logarithm of time. Initially $[\log (t)<1.7]$ the number of defects increases as the total droplet surface area increases. When 


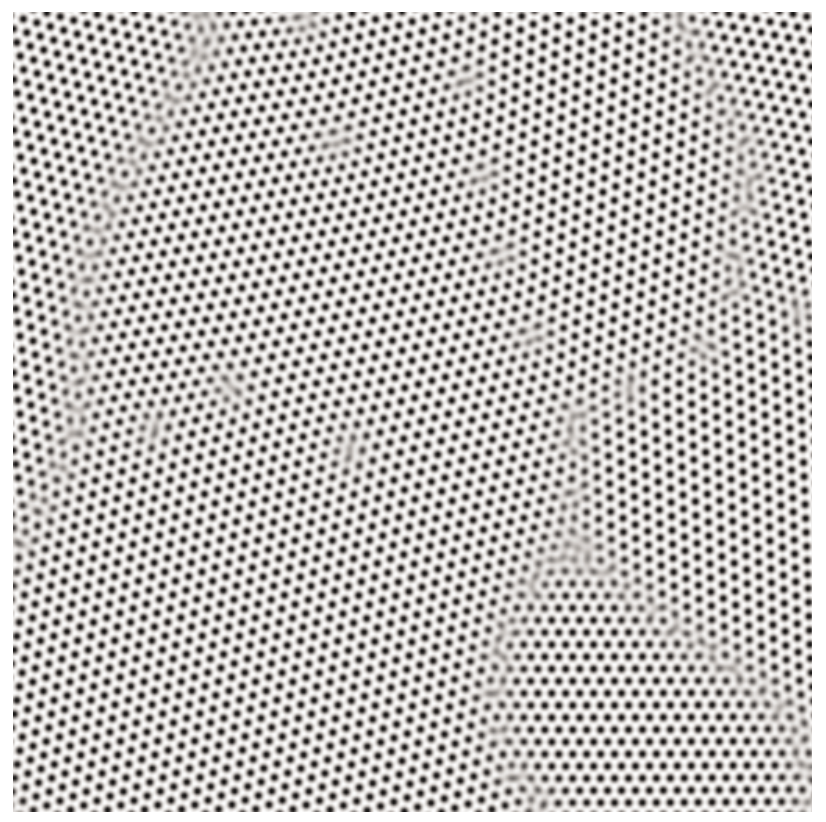

FIG. 2. Grain growth. Gray scale plot of the order parameter $\psi$ at $t / \tau_{D}=700$. This plot contains $1 / 64$ of the full simulations cell.

the droplets impinge, the number of defects begins to decrease as local rearrangements take place. At later times $\left[\log \left(t / \tau_{D}\right)>2.0\right]$, the grain boundaries evolve at a very slow rate. In these simulations, it is found that the number of defects decreases logarithmically at late times. The slow growth is distinctly different from foams and froths, where growth is mainly determined by the motion of triple junctions and curvature relaxation, and cannot be attributed to impurity pinning [20] since these simulations were conducted for a pure material.

The inclusion of elastic and plastic deformations allows the study of morphological instabilities in epitaxial growth. When a film grows on a bulk material that has a different lattice constant, the film can become corrugated ("buckle") in an attempt to relieve the strain [1]. The buckling of the surface relieves the strain in some regions but increases the strain in others. At these locations dislocations eventually nucleate. The critical height, $H_{c}$, at which these nucleate can be approximated by the Matthews and Blakeslee equation [3] which has the functional form, $H_{c}=H_{o}\left[1+\ln \left(H_{c} / b\right)\right] / \mathcal{E}$, where $H_{o}$ is a constant and $\mathcal{E}$ is the mismatch between the film and bulk lattice parameters; i.e., $\mathcal{E}=\left(a_{\text {film }}-a_{\text {bulk }}\right) / a_{\text {bulk }}$, where $a$ is a lattice constant.

To study these phenomena, a thin film with $q_{o}=q_{f}$ was grown on a bulk sample $\left(q_{o}=1\right)$ with the parameters $(\epsilon, \bar{\psi})=(1 / 4,1 / 4)$ for various values of $q_{f}$. The system had a width of $8192 \Delta x$ and a height of $1024 \Delta x$. A small portion of a sample simulation is shown in Fig. 3 for a lattice mismatch of $6.4 \%$, [i.e., $\mathcal{E}=\left(2 \pi / q_{f}-\right.$ $\left.\left.2 \pi / q_{o}\right) /\left(2 \pi / q_{o}\right)=0.064\right]$. The buckling phenomena are shown in Fig. $3 \mathrm{a}$ and the nucleation of dislocations in
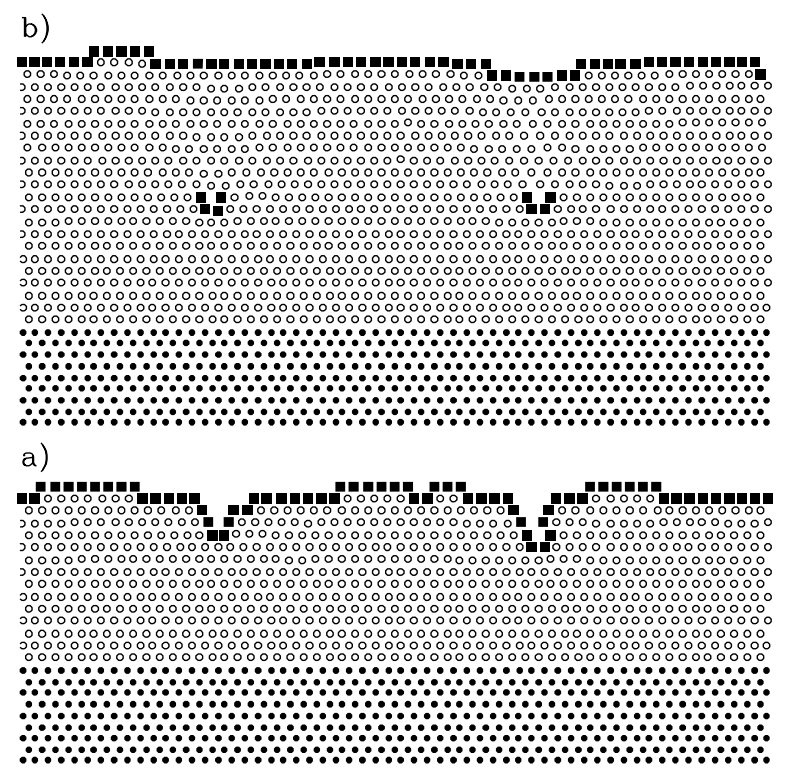

FIG. 3. Epitaxial growth. In this figure, the film and bulk particles (defined as local maxima of $\psi$ ) are plotted as open and closed circles, respectively. Defects are plotted as solid squares. Figures (a) and (b) are shown after an average of $\sim 13$ and $\sim 21$ layers have been grown, respectively. Figure (a) shows the buckling of the surface and (b) shows defects at the location of maximum buckle in (a). Figure (b) also shows that the creation of a defect leads to less buckling.

Fig. 3b. The numerical results for $H_{c}$ shown in Fig. 1d are consistent with the functional relationship proposed by Matthews and Blakeslee [3]. Figure 3 clearly shows that the nucleation of dislocations in epitaxial growth is highly correlated with surface buckling and must be included to obtain a quantitatively correct expression for the critical thickness. It is also noteworthy that the surface roughness decreases after dislocations appear, in accord with very recent rms surface roughening measurements on $\mathrm{SiGe} / \mathrm{Si}$ heterostructures [21].

The one component model described by Eqs. (1) and (2) does not support other metastable crystal phases and thus cannot be used to, for example, study structural phase transitions. However, it is straightforward to extend the model to include more than one kind of particle which can give rise to other metastable crystal phases. For example, a binary system can be easily modeled by a free energy of the form

$$
\begin{aligned}
\mathcal{F}=\int d \vec{r}\{ & \psi_{1}\left[\left(q_{1}^{2}+\nabla^{2}\right)^{2}-\epsilon_{1}\right] \psi_{1} / 2+\psi_{1}^{4} / 4 \\
& +\psi_{2}\left[\left(q_{2}^{2}+\nabla^{2}\right)^{2}-\epsilon_{2}\right] \psi_{2} / 2 \\
& \left.+\psi_{2}^{4} / 4+\alpha \psi_{1} \psi_{2}\right\}
\end{aligned}
$$

and the equations of motion, $\partial \psi_{1} / \partial t=\Gamma_{1} \nabla^{2} \delta \mathcal{F} / \delta \psi_{1}+$ $\eta_{1}$ and $\partial \psi_{2} / \partial t=\Gamma_{2} \nabla^{2} \delta \mathcal{F} / \delta \psi_{2}+\eta_{2}$, where $\alpha$ is a coupling constant. The properties (i.e., lattice constant, bulk compressibility, etc.) of the individual atoms are controlled by the parameters with subscripts 1 or 2 and by the average 


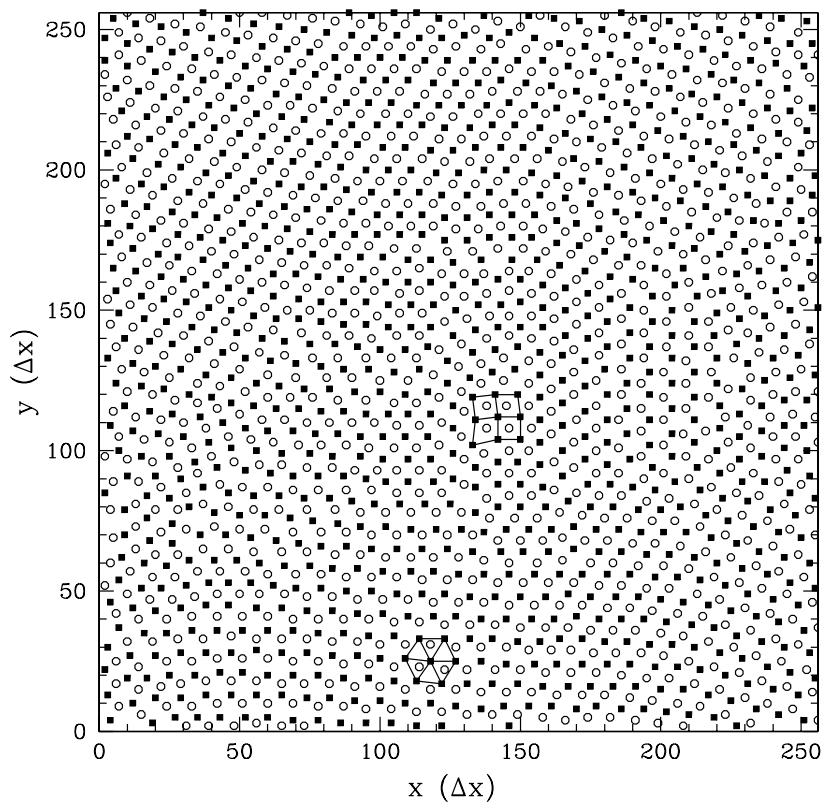

FIG. 4. Binary alloy. In this figure, the open circles and solid squares are the maxima of $\psi_{1}$ and $\psi_{2}$, respectively. Two different crystal structures have been highlighted by joining nearest neighbors of the same species.

value of $\psi_{1}$ and $\psi_{2}$. When an individual binary alloy droplet is grown, a hexagonal pattern typically emerges. However, when a random initial condition is used, the patterns usually contain more than one crystal phase since the energy of the hexagonal state is very close to a face centered cubic. One such configuration is shown in Fig. 4. Eventually, the system will evolve to a hexagonal state. Thus, the model can be used to study structural phase transitions.

In conclusion, Eqs. (1) and (2) model elastic and plastic behavior of crystals on atomic length scales and lead to simulations that are many orders of magnitude times faster than other approaches, such as molecular dynamics. For example, the current model can simulate one diffusion time in 1000 time steps, while molecular dynamics simulations would require $\sim 10^{9}$ time steps for a diffusion time of $1 \mu \mathrm{s}, \sim 10^{12}$ time steps for a diffusion time of $1 \mathrm{~ms}$ (appropriate for copper at $650{ }^{\circ} \mathrm{C}$ ), etc. Thus, this model should provide a useful tool for studying the crystallization phenomena.

This work was supported by Research Corporation Grant No. CC4787 (K.R.E.), and NSF-DMR Grant No. 0076054 (K. R.E.), the Academy of Finland (M.H.), the Natural Sciences and Engineering Research Council of Canada (M. G.), and le Fonds pour la Formation de Chercheurs et l'Aide à la Recherche du Québec (M. G.).
[1] R. J. Asaro and W. A. Tiller, Metall. Trans. 3, 1789 (1972).

[2] M. Grinfeld, Dokl. Akad. Nauk. SSSR 265, 836 (1982) [Sov. Phys. Dokl. 31, 831 (1986)]; Europhys. Lett. 22, 723 (1993).

[3] J. W. Matthews and A. E. Blakeslee, J. Cryst. Growth 27, 118 (1974); J. W. Matthews, J. Vac. Sci. Technol. 12, 126 (1975).

[4] P. C. Hohenberg and B. I. Halperin, Rev. Mod. Phys. 49, 435 (1977).

[5] B. Morin, K. R. Elder, M. Sutton, and M. Grant, Phys. Rev. Lett. 75, 2156 (1995).

[6] L.-Q. Chen and W. Yang, Phys. Rev. B 50, 15752 (1994).

[7] J. A. Warren, W. C. Carter, and R. Kobayashi, Physica (Amsterdam) 261A, 159 (1998); J. A. Warren, R. Kobayashi, and W. C. Carter, J. Cryst. Growth 211, 18 (2000); R. Kobayashi, J.A. Warren, and W. C. Carter, Physica (Amsterdam) 140D, 141 (2000).

[8] J. Müller and M. Grant, Phys. Rev. Lett. 82, 1736 (1999); M. Haataja, J. Müller, A. D. Rutenberg, and M. Grant, Phys. Rev. B 65, 035401 (2002).

[9] D. Orlikowski, C. Sagui, A. Somoza, and C. Roland, Phys. Rev. B 59, 8646 (1999).

[10] A. E. Jacobs, Phys. Rev. B 61, 6587 (2000).

[11] M. Sanati, A. Saxena, T. Lookman, and R. C. Albers, Phys. Rev. B 63, 224114 (2001).

[12] Y. U. Wang, Y.M. Jin, A. M. Cuitiño, and A. G. Khachaturyan, Appl. Phys. Lett. 78, 2324 (2001).

[13] A. Linhananta and D. E. Sullivan, Phys. Rev. E 57, 4547 (1998); I. I. Potemkin and S. V. Panyukov, Phys. Rev. E 57, 6902 (1998); J. Swift and P. C. Hohenberg, Phys. Rev. A 15, 319 (1977).

[14] C. Sagui and R. C. Desai, Phys. Rev. E 52, 2807 (1995).

[15] In this approximation the free energy is minimized when $A_{s}=2 \sqrt{3 \epsilon-9 \bar{\psi}^{2}} / 3, \quad q_{s}=q_{o}, \quad A_{h}=-4(\bar{\psi}+$ $\left.\sqrt{15 \epsilon-36 \bar{\psi}^{2}} / 3\right) / 5$, and $q_{h}=\sqrt{3} q_{o} / 2$.

[16] The change in energy for bulk, shear, and dilational deformations for an isotropic solid are, respectively, $\left(C_{11}+\right.$ $\left.C_{12}\right) \xi^{2},\left(C_{44} / 2\right) \xi^{2}$, and $\left(C_{11}-C_{12}\right) \xi^{2}$, where $\xi$ is the relative displacement for the different deformations. See, for example, F. Seitz, Modern Theory of Solids (McGraw-Hill, New York, 1940).

[17] See, for example, P. M. Chaikin and T. C. Lubensky, Principles of Condensed Matter Physics (Cambridge University Press, Cambridge, England, 1995); L. D. Landau and E.M. Lifshitz, Theory of Elasticity (ButterworthHeinemann, Oxford, 1998), 3rd ed.

[18] W. T. Read and W. Shockley, Phys. Rev. 78, 275 (1950).

[19] The diffusion time is $\tau_{D}=a^{2} / D$, where $a$ is the lattice constant, i.e., $a \approx 2 \pi /\left(\sqrt{3} q_{o} / 2\right)$.

[20] See, for example, G. Gottstein and L. S. Shvindlermann, Grain Boundary Migration in Metals: Thermodynamics, Kinetics, Applications (CRC Press, Boca Raton, Florida, 1999), p. 362.

[21] J. Gray, R. Hull, and J. Floro, Mater. Res. Soc. Symp. Proc. 696, N8.3.1 (2002). 